\title{
Notes d'entomologie marocaine
}

PAR

M. Antoine.

\section{XXXVIII ${ }^{1}$.-Les Crypticus du Maroc (Col. Tenebr.).}

Le genre Crypticus Latr. est extrêmement remarquable par son appareil copulateur.

La partie basilaire de la gaine paramérique (pièce basale de Sharp et Muir ${ }^{2}$, est normalement constituée, en forme de gouttière fermée ventralement par un velum membraneux, et bien distincte de la partie terminale. Celle-ci est formée de trois pièces: une dorsale et deux latérales.

Ces dernières, qui flanquent à droite et à gauche la pièce dorsale, s'infléchissent fortement en avant jusqu'à presque se toucher et se continuent à l'intérieur de la pièce basilaire, sous le velum, comme pour assurer la protection du canal éjaculateur, disposition qui atteint son maximum chez C. paradoxus Reitter. Elles sont toujours inégales, soit en longueur, soit en largeur, la droite plus développée que la gauche et constamment de forme différente.

La pièce dorsale se présente comme une tige plus ou moins déviée vers la gauche, plus ou moins tordue, à base élargie et bilobée, s'appuyant de chaque côté sur la pièce basilaire à laquelle elle est reliée au milieu par une zône membraneuse. Très fréquemment il existe un sillon longitudinal médian, témoin de l'origine double de la pièce, séparant les deux lobes basilaires et disparaissant vers l'apex. Ventralement, en écartant les pièces latérales, elle se montre (fig. Io, a) comme une sorte de gouttière largement ouverte en bas, rapidement rétrécie et fermée vers l'apex qui constitue une sorte de style plein; à la base on

1 Pour les notes I à XXXVII voir le Bull. S. Sc. Nat. Maroc, le Bull. de la S. Ent. de France (notes XXII-XXVIII-XXX) et le B. S. H. N. Afr. du Nord (XXXIII). La note XXXVII: «Adephaga terrestres du Maroc saharien» (B. Maroc, Xxir, I942, p. I50) a été publiée sans numéro d'ordre.

2 Sharp et MUIR: «The comparative anatomy of the male genital tube in Coleoptera». Trans. of the Ent. Soc., I9I2, p. 477.

Eos, XX, 1944. 
voit pénétrer le canal éjaculateur, ce qui m'avait amené à considérer cette pièce dorsale comme l'homologue du lobe médian de Sharp et Muir, dont la partie apicale, très développée et fortement chitinisée, se serait insinuée entre les deux paramères représentés par les pièces latérales.

Mais l'étude de l'appareil copulateur du C. corticeus Fairm. conduit à une interprétation toute différente. Dans cette pièce en effet, une vue du profil gauche (fig. I9, c) laisse voir, entre la pièce dorsale, conformée comme chez les autres Crypticus, et les pièces latérales (celles-ci soudées ventralement), une pièce interne supplémentaire, qui, disséquée, se montre (fig. 19, e) comme l'extrémité, libre et chitinisée sur sa face ventrale, du canal éjaculateur.

En conséquence la pièce dorsale est bien l'homologue de la partie apicale de la gaine paramérique telle qu'elle existe chez le type fondamental des Ténébrionides, mais profondément modifiée. La soudure du canal éjaculateur est une disposition secondaire qui n'est pas encore réalisée chez $C$. corticeus; elle a comme conséquence la fixité de cet organe, qui, normalement, coulisse à l'intérieur de la gaine. Quant aux pièces latérales, ce sont des formations supplémentaires correspondant vraisemblablement à un développement remarquable des lacinia.

Cette constitution très particulière de pénis, jointe à une asymétrie de l'organe parfois poussée jusqu'à la difformité fait des Crypticus un type très spécial digne d'être élevé au rang de sous-famille.

De cette sous-famille doivent être obligatoirement éliminés le petit genre myrmécophile Oochrotus Luc., et les Seriscius Motsch. (ces derniers considérés jusqu'à ce jour comme constituant un sous-genre de Crypticus), qui en diffèrent profondément par leur organe copulateur parfaitement symétrique. Je n'ai examiné à ce point de vue que l'O. unicolor Luc. et le S. adspersus s. sp. nebulosus Fairm. Mais, pour ce dernier genre, les croquis publiés par Koch ${ }^{1}$, si médiocres soient-ils, sont tout à fait démonstratifs.

La tribu des Crypticini comprend encore dans 'Winkler les deux petits genres Ellipsodes Woll. de Madère, et Cechenosternum Geb. d'Egypte. Ils me sont inconnus.

Les Crypticus du Maroc ont fait l'objet de tableaux de détermination de la part de Mr. A. Théry ${ }^{2}$. Ce travail, qui passe sous silence

$1 \mathrm{KocH}$ : 1937. «Tenebrioniden Lybiens». Publ. del Museo Ent. «Pietro Rossi». Duino, $\mathrm{n}^{\circ} 2$.

2 ThéRY: I933. «Notes d'ent. mar. et nord-afr.» (I Ième note). Bull. Maroc, XII, 1932, p. 195 . 
les caractères les plus intéressants, apparaît comme assez superficiel et ne m'a été d'aucune utilité.

Étude SYSTÉMATIQUE.

Les Crypticus du Maroc se séparent facilement en un certain nombre de groupes. Dans cette étude, géographiquement très limitée, seul le dernier sera élevé au rang de sous-genre.

\section{$I^{\text {er }}$ groupe.}

Caractérisé par ses stries bien creusées et grossièrement ponctuées. Une seule espèce marocaine:

I.-C. punctatolineatus Fairm. (fig. I).

Facile à distinguer par ses seul caractères externes.

L'organe copulateur est remarquable, d'une part, par sa partie basilaire anormalement élargie, munie d'une petite gouttière sur laquelle vient s'appuyer le canal éjaculateur, et, d'autre part, par la présence de deux bandes chitineuses flanquant à droite et à gauche le prolongement basilaire des pièces latérales; en réalité elles représentent la partie externe de celles-ci, avec lesquelles elles font corps et dont elles sont seulement séparées par un profond sillon. La pièce latérale gauche (fig. I, c) est en crochet à l'extrémité; la dorsale est comprimée en lame de sabreet je n'ai pu apercevoir l'orifice du canal éjaculateur.

Insecte des terres fortes des régions basses ou de médiocre altitude: Chaouia, plaine de Ber-Réchid, couloir Sud-Riffain (décrit de Fès); pas rare.

\section{zème groupe.}

Groupe subalpin ou alpin, composé d'espèces de taille médiocre our petite, glabres, peu sculptées, à caractères peu tranchés.

2.-C. azruensis Escalera (fig. 2).

L'organe copulateur est voisin de celui de punctatolineatus par son aspect général mais bien distinct par la conformation des différentes pièces. La pièce médiane présente un fort sillon dorsal (fig. 2, b); la partie basilaire des pièces latérales est simple, étroite, celle de gauche: est plus courte, à crochet terminal très accntué (fig. 2,c). 
Espèce largement répandue. Moyen-Atlas central ; Azrou (loc. typ.), Ifrane!, Timhadite!, et méridional: Arhbala! (Otin), Alemsid! (Otin).

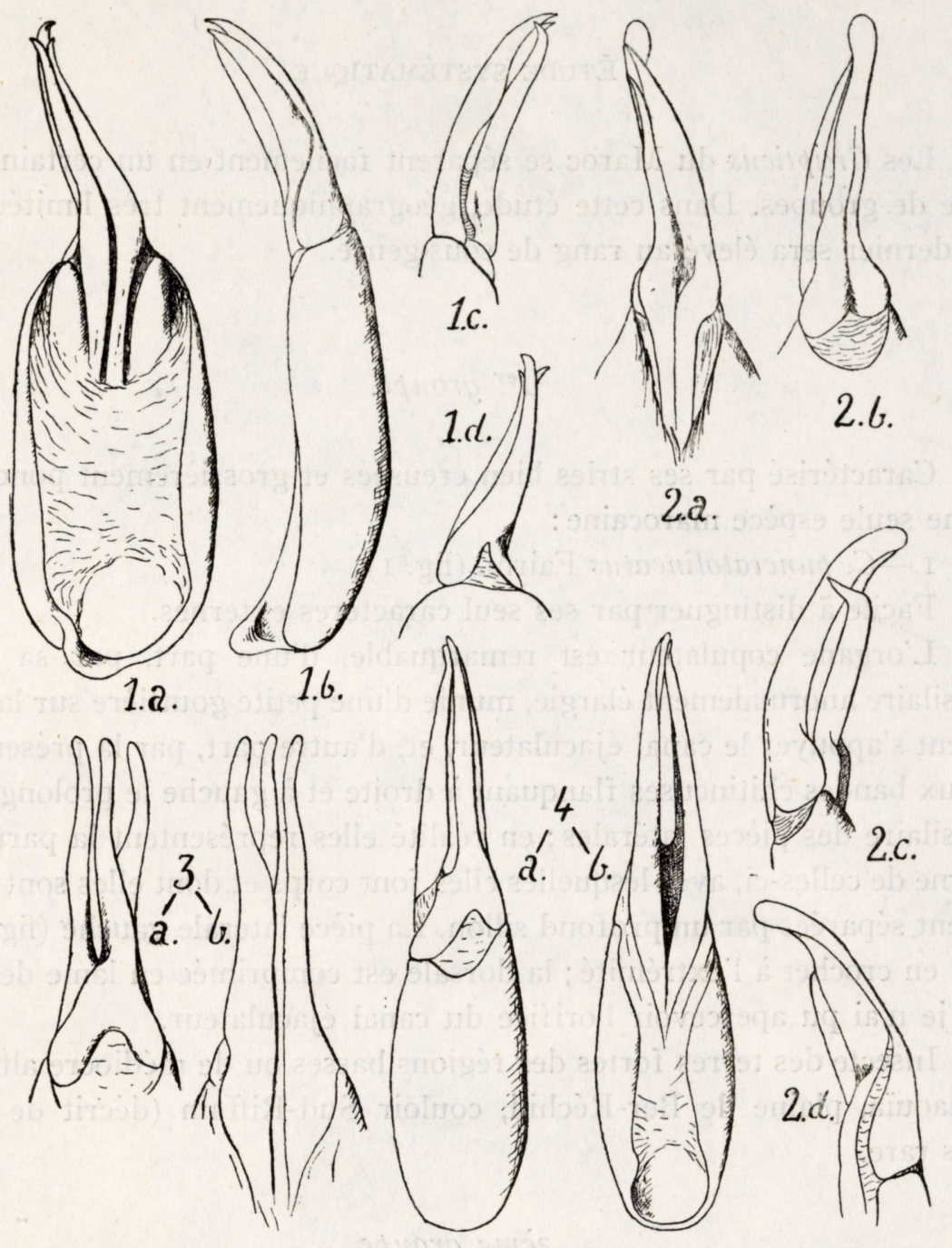

Figures I à 4. Premier et deuxième groupe--I. Crypticus punctatolineatus Fairm., de Casablanca: I $a$, organe copulateur, face ventrale; I $b$, profil droit; I $c$, profil gauche (région apicale); I $d$, face dorsale (région apicale).2. C. azruensis Escal., d'Azrou: $2 a$, apex de l'organe copulateur, face ventrale; $2 b$, le même face dorsale; $2 c$, le même profil gauche; $2 d$, le même profil droit.-3. C. Lecerfi Théry, du Tizi n'Test. Apex de l'organe copulateur en vue dorsale $(3 a)$ et ventrale $(3 b) .-4$. C. atlasicus Théry, d'Azrou. Organe copulateur en vue dorsale $(3 a)$ et ventrale $(3 b)$.

Aussi dans le Grand-Atlas: massif du Rhat! de I700 à $2400 \mathrm{~m}$. Les exemplaires de cette dernière localité sont dans l'ensemble plus fortement striés que ceux du Moyen-Atlas. 


\section{3.-C. Lecerfi Théry (fig. 3).}

Très caractérisé dans le groupe par ses antennes grêles et sa ponctuation prothoracique médiocre, mais dense et profonde. J'en possède un couple de la localité typique: Tizi n'Test!

L'appareil copulateur se met à part la gracilité des trois pièces api-cales qui se terminent au même niveau en divergeant.

4.-C. impunctatus Théry.

Décrit sur un seul exemplaire du Grand-Atlas (col. de Tarzeft). Je ne le connais pas; Théry, qui le déclare très ressemblant au dcctylispinus Mars., ne paraît pas avoir remarqué la structure toute spéciale des protarses de ce dernier; toutefois il a dû examiner sérieusement sa nouvelle espèce et ce caractère ne lui aurait certainement pas échappé s'il avait existé.

Par sa faible sculpture et son habitat alpin cette espèce me paraît devoir prendre place ici.

5.-C. atlasicus Théry (fig. 4).

C. atlasicus s. sp. culminicola Ant.

Petite espèce à voussure latérale forte, très brillante.

L'appareil copulateur est assez peu asymétrique, sa partie basilaire est proportionnellement beaucoup moins développée que chez les précédentes espèces; comme chez Lecerfi les trois pièces apicales sont subégales, mais elles sont étroitement rapprochées à l'extrémité.

Décrit d'Arhbalou n'Serdane dans le Moyen-Atlas méridional. Je le possède de la localité voisine d'Alemsid!, du Moyen-Atlas central: Azrou!, Ifrane!, Timhadite!, Aguelmane Sidi Ali!, et du Grand-Atlas: plateau des Lacs! (Otin). Les exemplaires de cette dernière localité sont de taille plus avantageuse.

Le C. culminicola, que je possède en nombre du Jbel Rhat, a le même organe copulateur et n'en diffère que par des caractères externes. Il doit être considéré comme une race d'atlcisicus.

\section{zème groupe.}

Ce groupe est caracterisé avant tout par l'élargissement, au niveau des épaules, de la gouttière marginale des élytres, et, accesoirement, par la taille moyenne ou grande, le corselet franchement plus large à la base que la base des élytres, ses téguments plus ou moins mats, à pubescence nulle ou réduite à des soies raides, extrêmement courtes et dressées sortant des points élytraux. 
Ce sont des insectes alpins, exclusivement marocains, descendant vraisemblablement d'un type ancestral qui aurait colonisé très anciennement la montagne marocaine et qui se serait scindé en un certain nombre de formes, extraordinairement voisines les unes des autres à ne considérer que la morphologie externe, mais que l'examen du pénis révèle plus ou moins profondément dissociées. L'isolement des massifs montagneux a vraisemblablement joué un rôle dans cette ségrégation; toutefois la présence simultanée, en certains points privilégiés (massif du Tachdirt par exemple) de plusieurs types spécifiques demeure, dans cette hypothèse, assez difficile à expliquer, à moins d'admettre une extension ultérieure des espèces nouvellement formées.

6.-C. petraeus Escalera (fig. 5).

Il se met tout à fait à part dans le groupe par son prosternum dont les flancs sont plans sur leur moitié antérieure et rejoignent le bord latéral sans former de gouttière. De plus, ses antennes sont courtes, tous les articles, à partir du 4ème, au moins aussi larges que longs, le corselet est peu transversal, très peu rétréci vers l'avant en courbe peu accentuée, avec les flancs fortement déclives. C'€st le plus petit du groupe et celui chez lequel l'élargissement de la gouttière élytrale est le moins accentué.

L'organe copulateur, vu par la face ventrale, est peu asymétrique; de profil, il est très arqué; la pièce latérale droite est beaucoup plus développée que la gauche.

Décrit du Tizi-n'Test (circa 2200 m.), d'où j'en possède un cotype que je dois à la générosité de Mr. de la Escalera; retrouvé au Tachdirt! (Schramm) vers $2700 \mathrm{~m}$.

7.-C. faucium Ant. (fig. 6).

Cette espèce a été décrite par comparaison avec le major Ant. que je considérais à tort à cette époque comme représentant le véritable Arandai Escal. Aussi en donnerai-je une courte diagnose.

Long. 7-8 $\mathrm{mm}$.

Tête en arc régulier, finement ponctuée. Corselet transversal, I,7 fois aussi large que long, le maximum de largeur à la base ou très près de la base, très rétréci vers l'avant en courbe régulière, les angles antérieurs très obtus, arrondis, les postérieurs droits, émoussés. Dessus convexe, avec les déclivités latérales assez fortes, à ponctuation un peu plus fine que celle de la tête et médiocrement dense; il existe une impression subpunctiforme de chaque côté, à égale distance du milieu et du bord latéral, et parfois aussi une autre dans la région des angles postérieurs. Elytres courts, convexes, avec une étroite impression trans- 
versale tout le long de leur extrême base, les stries externes approfondies à ce niveau. Lignes de points striales fines mais nettes, intervalles à ponctuation très fine assez dense. Antennes courtes, le zème article entre I f. I/ 2 et I f. $2 / 3$ aussi long que large, les suivants non cylindriques, le 4ème moins d'une fois et demie aussi long que large, le 5 ème un peu plus court, les 6ème, 7ème, 8ème et gème plus larges que longs, de plus en plus transversaux. Flancs du prosternum excavés, assez légèrement en avant, profondément en arrière.

Pénis très voisin de celui de petraeus, moins arqué, et, vu de profil, dépourvu de constriction préapicale.

Cette espèce paraît assez localisée. Grand-Atlas central: région du col de Tichka (types), Télouèt!, Tizi-n’Télouèt!, Jbel Aouljdid! Les exemplaires de cette dernière localité plus plats et un peu plus étroits que le type, ressemblent beaucoup au suivant.

8.-C. tachdirtensis nov. sp. (fig. 7).

Long. 7-8,25 $\mathrm{mm}$.

Extrêmement ressemblant au précédent; cependant un peu moins convexe, ce caractère surtout appréciable pour les élytres qui sont en outre un soupçon plus longs. Les antennes sont un feu plus épaisses, le 3ème article à peine une fois et demie aussi long que large, le 4ème presque isodiamétrique, les 5 ème et suivants de plus en plus transversaux; les gouttières latérales du prosternum sont plus creusé:s en avant que chez faucium. Enfin les $\hat{o}$ ô ont les métatibias légèrement incurvés en dehors.

L'appareil copulateur est bien particulier. Vues de face (fig. 7, a) les pièces latérales sont arquées, et, comme les branches d'une pince, viennent saisir la pièce médiane à une certaine distance de son extrémité ; celle de gauche, vue de profil (fig. $7, d$ ), est curieusement tordue en zig-zag; la pièce médiane présente un sillon basal dorsal et une protubérance ventrale sur laquelle se termine la gouttière ventrale.

L o c a 1 i té . - Localisé dans de massif du Likoumt, entre 2000 et $3000 \mathrm{~m}$. et même au dessus: Tizi-n’Tachdirt! (Schramm), haut assif Azourei!, Tizi Likoumt!, Tizi Ouanoums (Schramm), lac d'Ifni!

9.-C. rhaticus nov. sp. (fig. 8).

Long. 7-8,5 mm.

Dessus assez convexe ; tête en demi-hexagone, antennes assez épaisses, le zème article un peu plus d'une fois et demie aussi long que large, le 4ème une fois un quart, le 5ème à peine plus long que large, le suivant isodiamétrique, les derniers de plus en plus transversaux. Côtés du corselet rectilignes sur leur moitié postérieure, le plus souvent pa- 


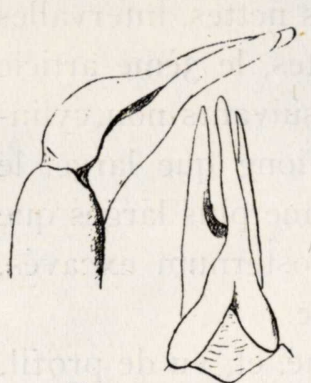

5..
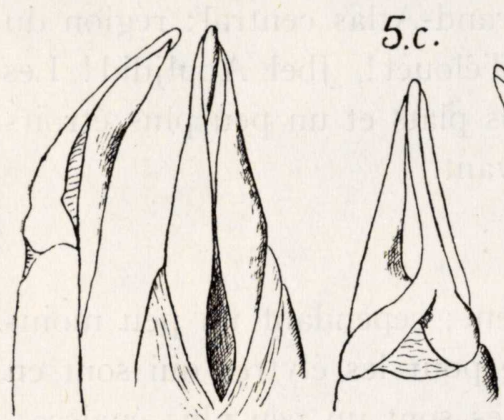

6.a. 6.6.

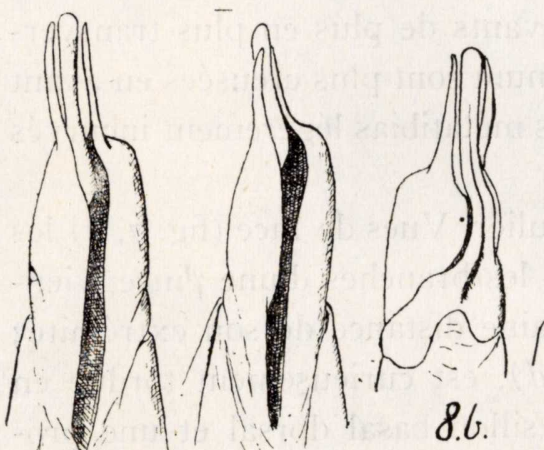

$8 . a$

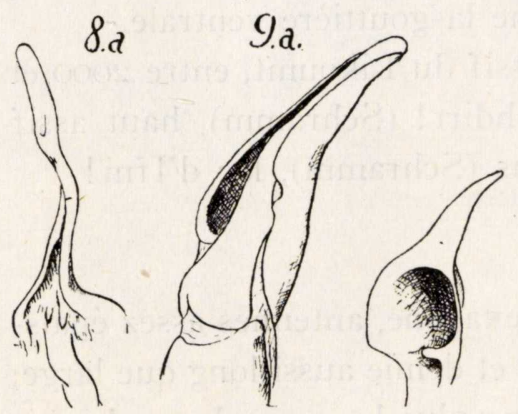

10.2. 10.6

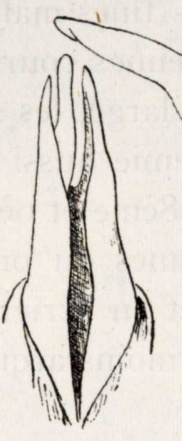

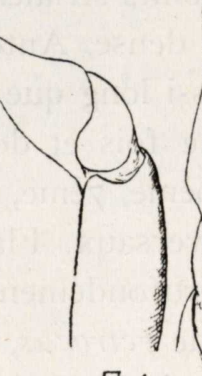

5.d.
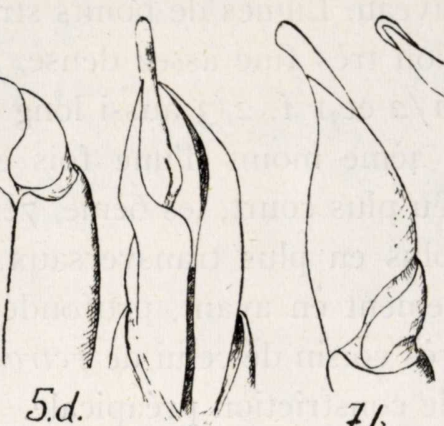

7.2.
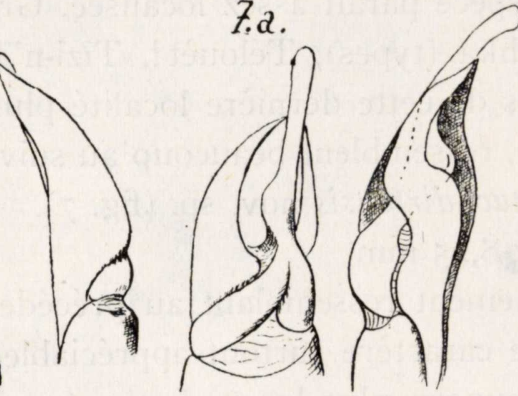

P

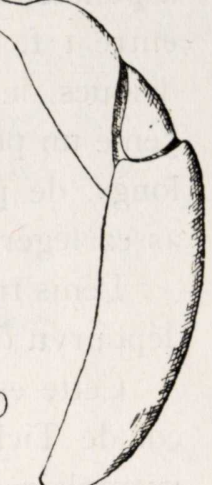

G.d. 7.c. 7.d.

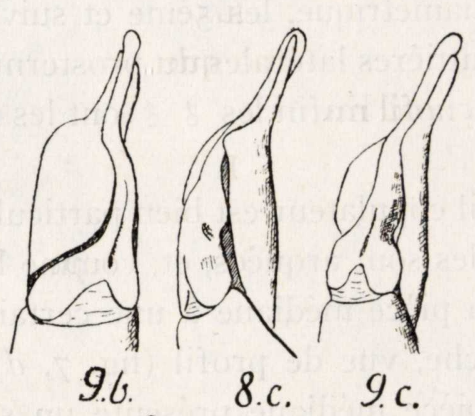

8d.
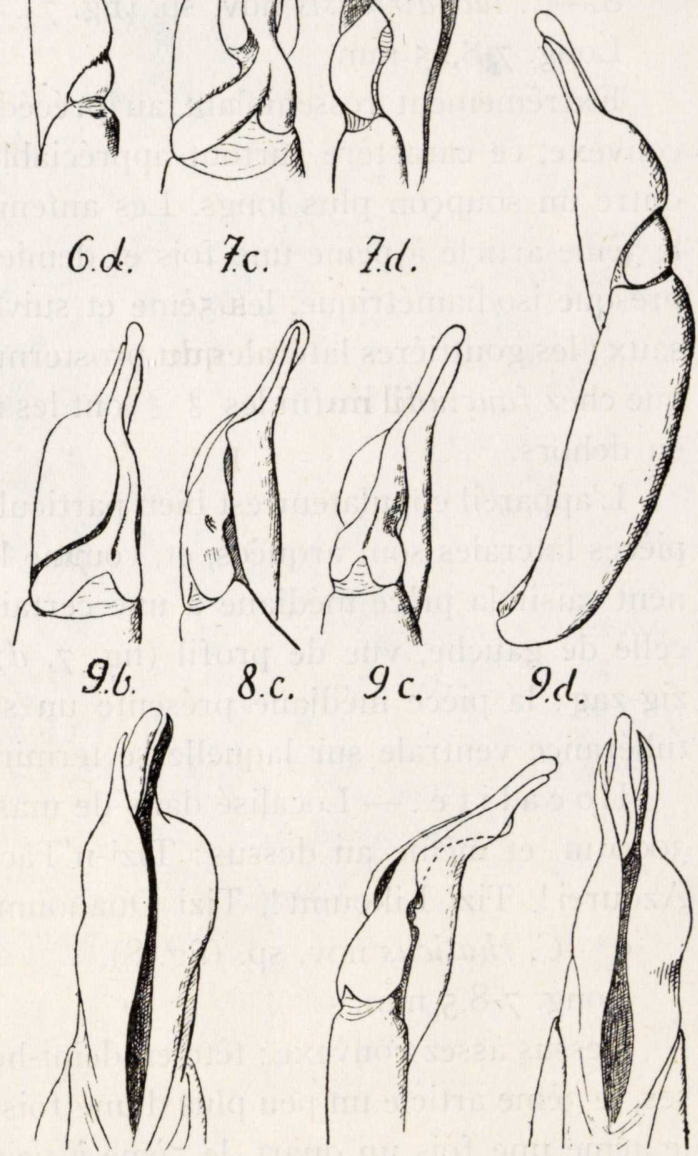

10.d.

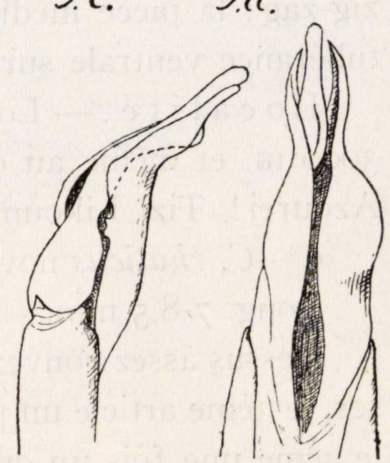

11. 11. 
rallèles mais parfois très légèrement rétrécis, ou vers l'avant, ou même vers l'arrière, la courbure antérieure ne commençant qu'à partir du milieu; méplat des angles postérieurs nul ou à peine indiqué. En dessous, la gouttière marginale du prosternum est très accentuée, même en avant. Pas de caractères sexuels secondaires.

Appareil copulateur présentant un rétrécissement brutal des pièces latérales avant l'apex, celle de gauche fortement anguleuse du côté dorsal; la pièce médiane est fortement sillonnée sur le dos. De profil, l’organe est assez arqué.

L o c a 1 i té . - Grand-Atlas central: Jbel Rhat!

Tachdirtensis, qui lui ressemble beaucoup est un peu plus déprimé avec les métatibias faiblement arqués chez le ô. Faucium est en général plus court, plus convexe, la gouttière latérale du prosternum est moins profonde en avant. Ces deux espèces ont d'ailleurs le pénis tout autrement conformé.

I0.-C. iblanensis nov. sp. (fig. 9).

Long. $7,5 \mathrm{~mm}$.

Diffère du précédent par son corselet à maximum de largeur tout à fait à la base, les côtés peu arqués, se rétrécissant régulièrement vers l'avant; les antennes sont encore plus épaisses, le 4ème article aussi large que long, les suivants de plus en plus transversaux.

Quoique de même type que celui de rhaticus le pénis est bien distinct par son profil moins arqué, la pièce médiane un peu plus tordue et, vue ventralement, à apex plus dévié vers la gauche, la pièce latérale gauche moins anguleuse, etc.

L o c a lité . - Moyen-Atlas septentrional: Bou-Iblane!, I ex.

\section{Explication des figures 5 à 11.}

Figures 5 à II. Troisième groupe.-5. C. petraeus Escal., du Tizi n'Tachdirt. Région apicale de l'organe copulateur: $5 a$, profil gauche; $5 b$, face dorsale; $5 c$, face ventrale; $5 d$, profil droit.-6. C. faucium Ant., du col de Tichka. Région apicale de l'organe copulateur: $6 a$, profil gauche; $6 b$, face ventrale; $6 c$, face dorsale; $6 d$, profil droit.-7. C. tachdirtensis Ant., du Tachdirt. Région apicale de l'organe copulateur: $7 a$, face ventrale; $7 b$, profil droit; $7 c$, face dorsale; $7 d$, profil gauche. -8 . C . rhaticus Ant., du Jebel Rhat. $8 a$, apex de l'organe copulateur vue ventrale; $8 b$, le même vue dorsale; $8 c$, le même profil gauche; $8 d$, l'organe entier profil droit.- 9. C. iblanensis Ant., du Bou Iblane. Même legende que pour le précédent; les croquis ont été intercalés pour permettre une comparaison plus facile.-Io. C. major Ant., du Tizi Ouchedden. Organe copulateur: Io $a$, pièce médiane isolée, face ventrale; Io $b$, région apicale profil gauche; Io $c$, pièce latérale droite vue par sa face interne; ro $d$, région apicale vue par la face ventrale-II. C. major s. sp. gundaficus Ant., du Tizi n'Test. Région apicale de l'organe copulateur: profil gauche: iा $a$; face ventrale: iा $b$. 
O b s e rvations. - Dans ce genre à organe copulateur compliqué et probablement en pleine évolution, on ne saurait affirmer que les différences réelles relevées à ce point de vùe entre l'insecte du BouIblane et celui du Rhat soient aussi décisives que des différences plus minimes, telles qu'on en peut signaler dans des genres à morphologie péniale plus uniforme (Pachychila par exemple). Mais alors que les cinq ou six autres espèces du groupe sont cantonnées dans une région du Grand-Atlas qui, en longueur, atteint à peine $200 \mathrm{~km}$., celle-ci se trouve isolée à quelque $300 \mathrm{~km}$. au Nord-Est et dans un autre massif montagneux, et ceci vient renforcer celà.

II.-C. major nov. sp. (fig. IO).

Long. 8-I I mm.

Quoique ressemblant aux précédents, facile à distinguer, au moins dans sa forme typique, par son facies; plus grand, plus plat, les côtés du corselet plus regulièrement arqués avec le maximum de largeur au tiers ou au quart postérieur, rétréci en avant et en arrière, en conséquence les angles postérieurs sont plus obtus et plus arrondis, vus d'en haut, nettement explanés, cet aplanissement parfois continué le long des côtés. Les antennes sont moins épaisses, le zème article 2 fois aussi long que large, le 4ème une fois un tiers, le 5 ème un peu plus long que large, les 6ème, 7ème, 8ème et gème aussi longs que larges, le ıoème transversal. Prosternum comme chez les précédents.

Appareil copulateur très à part, remarquable par la grosse boursouflure préapicale de la pièce latérale droite; la pièce médiane est dépourvue de sillon dorsal.

L o c a 1 i té. - Grand-Atlas central, région des Goundafa: TiziOucheddène! (types), et région des Glaoua: Tizi-n'Tachdirt! (Schramm), haut assif Azourei! ; entre 2000 et $3000 \mathrm{~m}$.

Les exemplaires du Tachdirt ont parfois les méplats prothoraciques peu accentués, ils se distinguent néanmoins facilement du tachdirtensis par leurs antennes visiblement plus grêles.

S. sp. gundaficus nov. subsp. (fig. II).

Bien distinct du type par ses dimensions plus faibles: $6,5-8 \mathrm{~mm}$., le corselet à côtés moins arqués, le maximum de largeur plus près de la base, par suite moins rétréci en avant et en arrière; les méplats sont moins accusés ce qui le fait plus ressemblant aux espèces précédentes qu'au major. Souvent le tégument est d'un soyeux mat très particulier. Le pénis est trop voisin de celui de la forme type pour permettre une séparation spécifique, toutefois l'examen des figures montre des différences sensibles: forme plus coudée de la région apicale vue de profil, 
boursouflure préapicale de la pièce latérale droite plus faible, pièce gauche plus anguleuse vers l'arrière, etc.

Cette race semble spéciale à la région du Tizi-n’Test! (Schramm).

12.-C. Arandai Escalera.

Décrite sur un unique exemplaire recueilli dans le Grand-Atlas vers I880 par Aranda et de provenance exacte inconnue, cette espèce ne paraît pas avoir été retrouvée.

J'extrairai de la description les passages suivants:

«Antenas llegando a los dos tercios del protórax, con su tercer artejo notablemente menor que los cuarto y quinto reunidos; éstos y el sexto subcilíndricos y dos veces más largos que anchos; los séptimo, octavo, noveno y décimo algo más largos que anchos, trapezoidales ... Protórax muy convexo en el disco, aplanado en los márgenes, que son muy visibles mirado el insecto perpendicularmente.»

Ces caractères et en particulier la gracilité des antennes ne peuvent s'appliquer à aucune des formes que j'ai capturées ou vues des différentes régions du Grand-Atlas. L'Arandai du Tachdirt signalé par Peyerimhoff ${ }^{1}$ est ou le major ou le tachdirtensis ou même un mélange des deux. En l'absence du type que ni Peyerimhoff ni moi n'avons vu, cette assimilation ne peut être faite qu'en suspectant Escalera d'une grosse erreur d'observation, ce qui m'apparaît peu probable.

\section{4ème groupe.}

Ici prendront place quelques espèces fortement caractérisées d'une part, par la structure du premier article des protarses dont la sole inférieure est très développée (fig. I3), plus longe que la face supérieure et déborde plus ou moins ou dessous du zème article (ce prolongement varie beaucoup dans ses dimensions et parait, sans toutefois que ce caractère soit absolu, plus développé chez les ô ô que chez les 우 우), et, d'autre part, par la torsion autour de son grand axe présentée par la partie basilaire de l'organe copulateur (figs. I2 et I4).

I3. C. dactylispinus Marseul (fig. I2).

Décrit d'Oran et de Biskra; il est probable que seuls les individus oranais doivent être considérés comme typiques. Je leur rapporte un insecte assez abondant dans la vallée de la moyenne Moulouya: Ber-

1 Peyerimhoff: 1928. «Composition et origine présumée de la faune (Coleopt.) des hautes montagnes de la Berbérie. Soc. de Biogéographie, II. Cont. à l'étude du peuplement des Hautes montagnes. 
guent (Alluaud), Guercif! (Otin), Taourirt! (Otin), El-Guettaf, BouaSidi, Midelt (Schramm), Safsafate (Rotrou), et que Mr. Schramm et moi-même avons retrouvé beaucoup plus au sud à Sidi-Moktar entre Mogador et Marrakech. C'est un habitant des régions basses et subdésertiques.

Sous le nom de dactylispinus j'ai reçu jadis du Dr. Chobaut quatre individus de Gafsa (Tunisie) qui appartiennent à une tout autre espèce ${ }^{1}$. Les dactylispinus signalés de Lybie par $\mathrm{Koch}^{2}$ se rapportent soit à cette dernière espèce soit à autre chose.

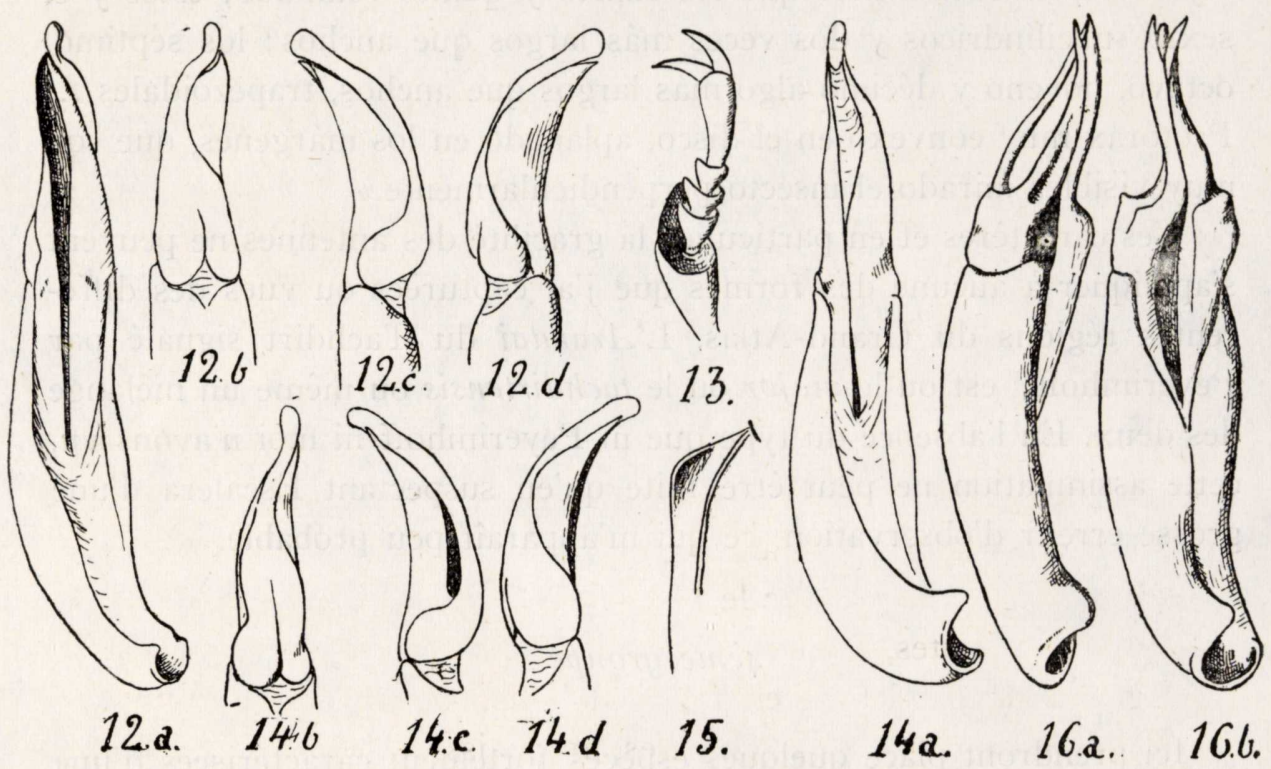

Figures I2 à I6. Quatrième groupe.-12. C. dactylispinus Mars., de Guercif. I2 $a$, organe copulateur, face ventrale; i2 $b$, région apicale, face dorsale; I2 $c$, la même, profil droit; I2 $d$, ia même, profil gauche.-I3. Protarse du même.-I4. C. Schrammi Ant., du Cap Rhir, I4 $a$, organe copulateur, face ventrale; I4 $b$, région apicale, face dorsale; I 4 , la même, profil droit; i $4 d$, la même, profil gauche.-I5. Faux epipleures du même, région humérale.-i6. $C$. capsianus Ant., de Gafsa. Organe copulateur, vue ventrale ( $16 b$ ) et profil gauche (I6a).

1 C. capsianus nov. sp.-Extrêmement voisin extérieurement de C. dactylispinus Mars, dont il ne diffère guère que par ses dimensions un peu plus fortes: $5,5-6,3 \mathrm{~mm}$., sa forme un peu plus étroite, les élytres visiblement plus longs, les stries élytrales plus distinctes et les articles 5-7 des antennes plus grêles. La coloration est moins franchement noire, plutôt brun de poix sombre. Mais l'ap̣pareil copulateur est extraordinairement différent et sans analogue dans toute la série des Crypticus. Toutefois comme dans le deux espèces marocaines la partie basilaire est tordue sur son axe (fig. I6).

Loc a 1 i té: Gafsa (Chobaut).

2 Косн: Op. cit., p. 199. 
I4.-C. Schrammi nov. sp. (fig. I4).

Extrêmement voisin du précédent. Même forme et même dimension, mais bien distinct à première vue par son corselet plus bombé, à côtés plus fortement arqués et à ponctuation beaucoup plus forte et plus serrée; en outre les épipleures sont plus élargis dans la région humérale, et, à ce niveau, nettement plus concaves (fig. I 5). Le pénis, de même type, est cependant bien différent par sa pièce médiane plus étroite et moins tordue à l'extrémité et les pièces latérales autrement conformées.

L o c a 1 i té . - Cap Rhir, 5 ex. recueillis par Mr. Schramm et par moi-même sur les sables de décomposition de la dune consolidée tout près de la mer; Agadir! I ex.

Sans doute vicariant méridional du précédent.

5ème groupe.

Je réunis ici, afin de ne pas trop multiplier les divisions, deux insectes qui, quoique très dissemblables, présentent deux caractères communs à savoir la pubescence du dessus assez développée, et, surtout, la forme de la gouttière latérale du prothorax, laquelle résulte du raccord progressif des marges avec le fin rebord redressé, tandis que, chez les espèces précédentes, les marges s'infléchissent brusquement en donnant une gouttière très étroite en forme de sillon ou mieux de rainure. Ce sont des formes de plaine, estivales, apparaissant vers la fin mai pour disparaître avec les premières pluies.

I5.-C. gibbulus Quensel (fig. I7).

Espèce très anciennement connue et qui paraît avoir une plus large répartition que la normale. Peut-être y aurait-il lieu de vérifier l'identité de l'organe copulateur d'individus de provenances diverses; chez tous mes exemplaires marocains il est remarquable par son aspect cylindracé; la partie basilaire, pas plus large que la partie apicale, est anormalement échancrée à la base ; la pièce latérale droite est mucronée et la pièce médiane est fortement infléchie à l'apex, presque hamiforme.

C'est le plus abondant de nos Crypticus indigènes depuis le Maroc oriental jusqu'à Agadir; il gagne même la zône subalpine: Azrou!, Télouèt!, flancs du Likoumt! et du Rhat! jusqu'à I $800 \mathrm{~m}$. environ.

I6.-C. paradoxus Reitter (fig. I8).

Très à part par son facies et son organe copulateur extrêmement évolué: les pièces latérales, énormes, constituent presque à elles seules 
toute la face ventrale, leur région apicale est concave dorsalement en forme d'oreilles, avec un bourrelet marginal externe; la pièce médiane est fortement déviée vers la gauche, le lobe basilaire gauche presque disparu.

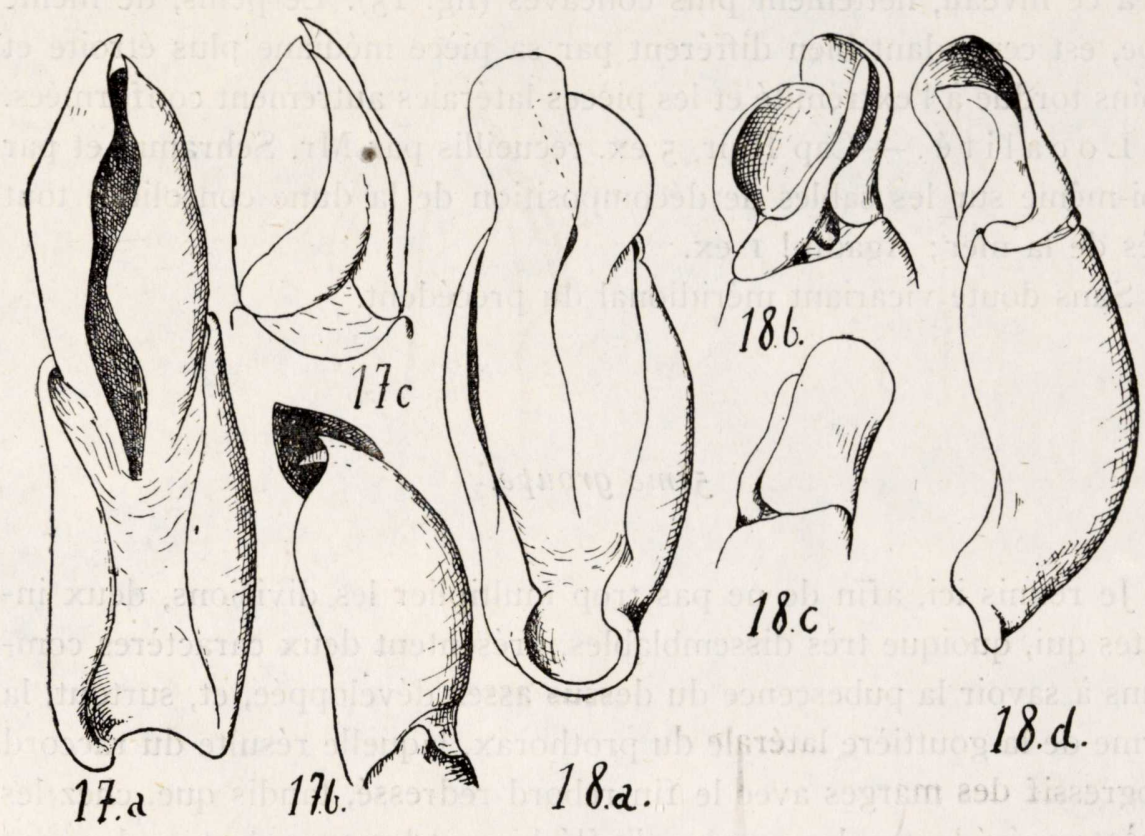

Figures I7 et I8. Cinquième groupe.-I7. C. gibbulus Quens., de Casablanca. I7 $a$, organe copulateur, vue ventrale; $17 b$, profil droit de la région apicale; I7 $c$, face dorsale de la même.-I8. C. paradoxus Reitter, de Casablanca. I $8 a$, organe copulateur, face ventrale; I $8 b$, région apicale, face dorsale; I $8 c$, la même, profil gauche; I8 $d$, profil droit, vue d'ensemble.

Je ne le connais que de la zône littorale ou sublittorale: Casablanca!, Zénata!, Médiouna!, Mazagan (Bleton!); assez rare.

Classé à tort dans le catalogue Winkler comme Seriscius.

Gème groupe: Crypticopsis s. g. nov.

type: C. corticeus Fairmaire.

Les deux insectes que je range dans ce nouveau sous-genre se distinguent des autres Crypticus par leur menton plan, dépourvu de fine carène longitudinale médiane, et par leurs métatibias coupés oblique- 
ment à leur extrémité; les tarses postérieurs sont très grêles comme chez les Seriscius. L'appareil copulateur est semblable à celui des autres Crypticus, mais la soudure de la pièce médiane (lobes latéraux de Sharp et Muir) avec la terminaison du canal éjaculateur (lobe médian des mêmes auteurs) n'est pas encore opérée et celui-ci est visible de profil, très nettement chez corticeus, beaucoup moins chez meridionalis.
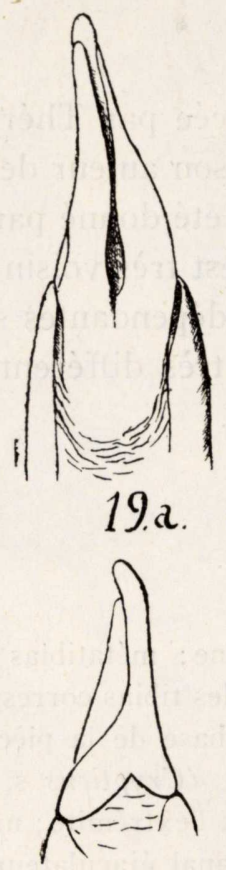

20.6.
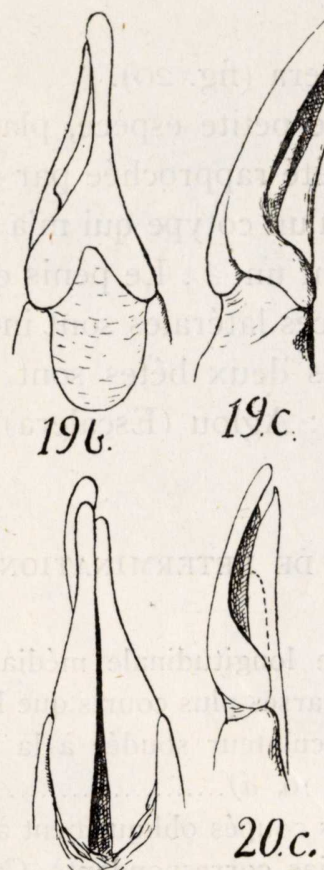

20.c.
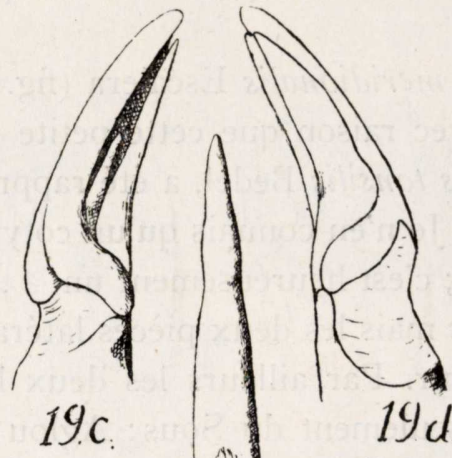

$19 d$.

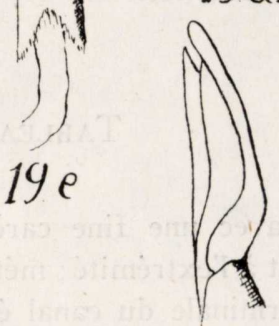

20.d.

Figures 19 et 20. S. g. Crypticopsis.-19. C. corticeus Fairm., de Casablanca. Ig $a$, région apicale de l'organe copulateur, face ventrale; Ig $b$, face dorsale; I9 $c$, profil gauche montrant entre la pièce dorsale et la pièce latérale gauche l'extrémité du tube éjaculateur; I9 $d$, profil droit; I9 $e$, extrémité isolée et fortement grossie du tube éjaculateur.-20. C. meridionalis Escal., d'Aglou. $20 a$, région apicale de l'organe copulateur, face ventrale; $20 b$, face dorsale; $20 c$, profil gauche; $20 \mathrm{~d}$, profil, droit.

Bien que le genre soit assez peu homogène dans l'ensemble, ces caractères me paraissent suffisants pour nécessiter l'isolement de ces deux formes.

Ce sont deux espèces littorales franchement sabulicoles et plus ou moins dépigmentées.

I7.-C. corticeus Fairmaire (fig. I9).

Se met tout à fait à part parmi les Crypticus marocains, par sa 
saillie prosternale très aiguë et longuement prolongée au delà des hanches antérieures.

L'organe copulateur présente une particularité intéressante: les pièces latérales, très élargies et rappelant celles de l'espèce précédente, sont soudées, sur leur moitié basilaire en une plaque unique.

Abondant dans les dunes dès le premier printemps, enterré aut pied des plantes (en particulier Euphorbia paralias), de Tanger à Agadir.

I8.-C. meridionalis Escalera (fig. 20).

C'est avec raison que cette petite espèce, placée par Théry à côté du Seriscius tonsilis Bedel, a été rapprochée par son auteur de l'espèce précédente. Je n'en connais qu'un cotype qui m’a été donné par Mr. de la Escalera; c'est heureusement un $\hat{o}$. Le pénis est très voisin de celui de corticeus mais les deux pièces latérales sont indépendantes sur toute leur longueur. Par ailleurs les deux bêtes sont très différentes.

Connu seulement du Sous: Aglou (Escalera).

\section{TABLEAU DE DETERMINATION.}

I. Menton avec une fine carène longitudinale médiane; métatibias tronqués: carrément à l'extrémité ; métatarses plus courts que les tibias correspondants. Partie terminale du canal éjaculateur soudée à la base de la pièce apicale de la gaine paramérique (fig. Io, a) .................... (Crypticus s. str.) 2

- Menton non caréné ; métatibias coupés obliquement à l'extrémité; métatarses grêles, aussi longs que les tibias correspondants. Canal éjaculateur normal, libre et chitinisé à son extrémité (fig. I9, $c$, etc.)......

(Crypticopsis) I7

\section{Sous-genre Crypticus s. str.}

2. Extrême bord du corselet plus ou moins brusquement déclive au dessus du fin rebord latéral en sorte que la gouttière marginale apparaît comme une fine rainure bien gravée; dessus pratiquement glabre.

- Extrême bord du corselet progressivement raccordé avec le rebord latéral, la gouttière marginale, par suite, mal délimitée du côté interne; dessus distinctement pubescent.

3. Premier article des protarses normalement conformé. Partie basilaire de l'organe copulateur, vue de face, symétrique ou à peine un peu gauchie à l'apex.

- Premier article des protarses coupé obliquement en cornet à l'extrémité (fig. I3), la face inférieure plus développée et plus ou moins prolongée au dessous des articles suivants. Partie basilaire de l'organe copulateur tordue autour de son axe à presque $45^{\circ}$ (fig. I $2, a$ ) 
4. Gouttière marginale des élytres linéaire sur toute sa longueur.............. 5

- Gouttière marginale des élytres plus ou moins élargie en avant au niveau des épaules.

5. Stries, surtout externes, profondément tracées et toutes jalonnées de gros points. Insecte de plaine, assez svelte, brun, de poix ou roux foncé. 5,5-7 mm. punctatolineatus Frm.

- Pas de stries, seulement des points fins, alignés, qui peuvent même manquer. Insectes alpins ou subalpins, courts, noirs ou brun de poix foncé.......... 6

6. Lignes de points striales bien nettes. Pièce latérale gauche du pénis plus courte que la droite, coudée à angle droit et acuminée à l'extrémité, pièce médiane profondément sillonnée sur sa face dorsale (fig. 2,b). Assez court et assez convexe. $5,5-6,5 \mathrm{~mm}$. ............................... azruensis Esc.

- Lignes de points striales indistinctes ou nulles. Pénis, là où il est connu, autrement conformé.

7. Pronotum à ponctuation médiocre mais profonde et dense, subconfluente latéralement. Antennes assez grêles à articles 4-7 au moins aussi longs que larges. Piéces latérales du pénis égales, divergentes à l'apex. Insecte mat, assez étroit, peu convexe. $5,5-6,3 \mathrm{~mm}$. ...................... Lecerfi Théry.

- Pronotum à ponctuation très fine et assez espacée. Antennes épaisses, à articles 4-7 aussi larges ou plus larges que longs ............................. 8

8. Ponctuation élytrale encore plus fine que celle du corselet, celui-ci grand, transversal, à côtés régulièrement arqués; articles des antennes, à partir du 4éme, aussi longs que larges. $5,8 \mathrm{~mm}$. ............... impunctatus Théry.

- Ponctuatioin élytrale plus forte que ;celle du corselet, celui-ci plus étroit, à côtés peu arqués; articles des antennes 8-9-10 fortement transversaux.

a) Corselet plus court, plus large à la base que la base des élytres, forme générale plus trapue. $4-6 \mathrm{~mm}$. .............................. atlasicus Théry.

b) Corselet plus étroit, aussi large à la base que la base des élytres, forme générale un peu plus svelte. 3,9-4,2 mm. .......... s. sp. culminicola Ant.

9. Antennes assez épaisses, les articles 4-5 au plus une fois et demie aussi longs que larges, les suivants au plus isodiamétriques .......................... Io

- Antennes grêles, les articles 4-5 deux fois aussi longs que larges, les suivants jusqu'au Ioème plus longs que larges. Angles postérieurs du corselet explanés, les côtés bien arqués. $8 \mathrm{~mm}$. ............................. Arandai Esc.

Io. Flancs du prosternum dépourvus de gouttière marginale sur leur moitié antérieure où ils se raccordent «de plano» avec les bords du pronotum. Corselet peu transversal à côtés peu arqués, peu rétrécis en avant. Antennes à articles très courts. Pénis, vu ventralement, subsymétrique (fig. $5, c$ ). 4,5-6 mm. petraeus Esc.

- Flancs du prosternum plus ou moins en gouttière sur leur moitié antérieure. Corselet plus transversal; antennes plus grêles. 6,5-I I mm. II

II. Pénis, vu par sa face ventrale, subsymétrique (fig. $6, b$ ). Gouttière marginale du prosternum peu accentuée en avant, déclivités latérales du pronotum assez fortes, forme générale plus convexe. 7-8 mm. .......... faucium Ant.

- Pénis fortement asymétrique. Gouttière margina'e du prosternum plus accentuée. Dessus moins convexe.

12. Pièces latérales du pénis, vues ventralement (fig. $7, a$ ) arquées à l'apex, enserrant comme une pince la pièce médiane dont l'extrémité les dépasse 
longuement. Pièce latérale gauche en zig-zag. Métatibias des $\hat{o} \hat{o}$ légèrement arqués en dehors. $7-8,25 \mathrm{~mm}$. tachdirtensis Ant.

- Pièces latérales du pénis, vues ventralement, brutalement rétrécies avant l'apex et ensuite appliquées plus ou moins étroitement sur l'extrémité de la pièce médiane qui les dépasse à peine. Pièce latérale gauche autrement conformée. Métatibias des ô ô rectilignes.

I3. Flanc externe de la pièce latérale droite, avant le rétrécissement apical, parallèle au grand axe, nullement tuméfiée (figs. 8 et $9 a$ ); face dorsale de la pièce médiane sillonnée (figs. 8 et $9, b$ ). Antennes plus épaisses. zème article I fois et demie aussi long que large, les articles 6-Io tranversaux; méplat des angles postérieurs du corselet nul ou peu marqué.

- Flanc externe de la pièce latérale droite fortement boursouflé (fig. Io, d); face dorsale de la pièce médiane sans sillon. zème article des antennes deux fois aussi long que large, le roème seul transverse.

a) Côtés du corselet plus arqués, nettement rétrécis vers l'arrière, méplat des angles postérieurs très net. Boursouflure de la pièce droite forte, bulleuse (fig. Io, d), base de la pièce médiane plus transversale. 8-II mm. ........................................................... major Ant.

b) Côtés du corselet moins arqués, le maximum de largeur plus vers la base, par suite moins rétrécis en arrière, méplat des angles postérieurs moins net. Pièce latérale droite moins tuméfiée (fig. II, b), base de la pièce médiane plus étirée longitudinalement. $6,5-8 \mathrm{~mm}$.

s. sp. gundaficus Ant.

14. Côtés du corselet parallèles ou subparallèles sur leur moitié basilaire, antennes un peu moins épaisses. Pénis, vu de profil, plus arqué (fig. $8, d$ ); pièce latérale gauche plus large, anguleuse du côté dorsal (fig. 8, c). Pièce médiane moins tordue (fig. 8, b). Espèce du Grand-Atlas. 7-8,5 mm.

rhaticus Ant.

- Côtés du corselet rétrécis vers l'avant dès la base. Antennes très épaisses. Pénis, vu de profil, moins arqué (fig. $9, d$ ); pièce latérale gauche étroite, non anguleuse (fig. 9, c), pièce médiane en vue dorsale fortement tordue (fig. 9, b). Espèce du Moyen-Atlas septentrional. 7,5 $\mathrm{mm}$.

iblanensis Ant.

15. Pronotum moins convexe, à ponctuation fine, assez espacée, ses côtés moins arqués; faux épipleures moins élargis et moins concaves au niveau des épaules. Pièce basilaire du pénis moins tordue (fig. I2, $a$ ), pièces apicales autrement conformés (fig. $\mathrm{I} 2, b, c, d) .5-6 \mathrm{~mm}$. ................ dactylispinus Mars.

- Corselet plus bombé, à ponctuation médiocre et dense, ses côtés plus arqués; faux épipleures plus élargis et plus concaves aux épaules. Pièce basilaire du pénis fortement tordue autour de son axe (fig. I4, a). pièces apicales autrement conformées (fig. I4, b, c, d) . 5-6 mm. ............. Schrammi Ant.

I6. Angles postérieurs du corselet droits, simplement émoussés à l'apex; séries de points striaux très nets; pubescence courte. Insecte svelte, noir peu brillant uniforme. $6,5-7,8 \mathrm{~mm}$. gibbulus Quens.

- Angles postérieurs du corselet très largement arrondis, points striaux indistincts, pubescence du dessus très longue. Petit insecte très court et très convexe, roux avec les intervalles pairs plus foncés. Organe copulateur très particulier (fig. I8). 3,2-4 mm. paradoxus Reitt. 
Sous-genre Crypticopsis.

17. Saillie prosternale très longue spiniforme. Pièces latérales du pénis soudées (fig. I9, a). Insecte très convexe à facies de Zophosis. 4,5-6 mm.

a) Corselet plus transversal. plus rétréci vers l'avant; forme plus courte. De Tanger ou Cap Cantin. corticeus Fairm.

b) Corselet plus long, moins rétréci vers l'avant, forme plus svelte. Safi!, Mogador. s. sp. proximus Esc.

- Saillie prosternale beaucoup plus courte. Pièces latérales du pénis non soudées (fig. 20, a). Insecte moyennement convexe, élytres à côtés parallèles sur leur première moitié. 3-5 $\mathrm{mm}$. meridionalis Esc.

\section{Conclusions.}

L'organe copulateur des Crypticus, dont l'étude fait en somme l'objet principal de ce travail, est extrêmement remarquable par le grand développement de deux pièces supplémentaires que j'ai appelées pièces ventrales, et qui correspondent vraisemblablement aux lacinia des Dendarini. Un autre caractère, des plus curieux, de cet organe, est la disparition de l'extrémité chitinisée du canal éjaculateur (lobe médian de Sharp et Muir), celui-ci se soude en effet à la base de la pièce que j'ai appelée médiane et que correspond à la partie terminale de la gaine paramérique des Ténébrionides de type normal. Toutefois, dans deux espèces, cette soudure n'est pas encore réalisée, ce qui m’a obligé à les isoler dans un sous-genre nouveau: Cryticopsis. Enfin, et ceci n'est pas le moins intéressant, il est fort asymétrique, cette asymétries allant parfois jusqu'à la difformité.

Cette structure très particulière nécessite l'élévation de la tribu au rang de sous-famille. De cette sous-famille devront être éliminés les Seriscius Motsch. et les Oochrotus Luc. La position des petits genres Ellipsodes Woll. et Cechenosternum Geb. reste à déterminer.

D'ailleurs d'une façon générale, le genre est assez peu homogène et, même dans cette étude limitée, j'ai pu facilement distinguer des groupes: dont quelques-uns devront probablement, dans une révision générale, être érigés en sous-genres.

Les Crypticus marocains, à l'exception des gibbulus et dactylispinus, sont tous endémiques, beaucoup, surtout parmi les espèces alpines, étant 
étroitement localisées. Une mention spéciale doit être faite à propos du groupe petraeus, qui, avec une morphologie externe très uniforme, présente une variabilité extraordinaire de l'appareil pénien.

Dans ce travail sont décrits un sous-genre nouveau, six espèces (dont une etrangère du Maroc) et une sous-espèce nouvelles. 